\title{
External validation of simplified out-of-hospital cardiac arrest and cardiac arrest hospital prognosis scores in a Japanese population: A multicentre retrospective cohort study
}

Keita Shibahashi ( $\square$ kshibahashi@yahoo.co.jp)

Tokyo Metropolitan Bokutoh Hospital https://orcid.org/0000-0001-5651-1449

Kazuhiro Sugiyama

Metropolitan Bokutoh Hospital

Yusuke Kuwahara

Tokyo Metropolitan Bokutoh Hospital

Takuto Ishida

Tokyo Metropolitan Bokutoh Hospita

Atsushi Sakurai

Nihon University School of Medicine

Nobuya Kitamura

Kimitsu Chuo Byoin

Takashi Tagami

Nippon Medical School Musashikosugi Hospital

Taka-aki Nakada

Chiba University Graduate School of Medicine

Munekazu Takeda

Tokyo Women's Medical University

Yuichi Hamabe

Tokyo Metropolitan Bokutoh Hospital

\section{Research}

Keywords: Out-of-hospital cardiac arrest, predictive score, risk score, validation, neurological outcomes

Posted Date: January 10th, 2020

DOI: https://doi.org/10.21203/rs.2.20595/v1

License: @ (i) This work is licensed under a Creative Commons Attribution 4.0 International License. Read Full License

Version of Record: A version of this preprint was published at Emergency Medicine Journal on July 21st, 2021. See the published version at https://doi.org/10.1136/emermed-2020-210103. 


\section{Abstract}

Background

Out-of-hospital cardiac arrest (OHCA) is a global medical problem. The newly-developed simplified out-of-hospital cardiac arrest (sOHCA) and cardiac arrest hospital prognosis (sCAHP) scores used for prognostication of patients admitted alive have not been validated externally. This study was, thus, conducted to externally validate sOHCA and SCAHP scores in a Japanese population.

Methods

Adult patients resuscitated and admitted to hospitals after intrinsic OHCA ( $n=2,428$, age $\geq 18$ years) were selected from a prospectively collected Japanese database (January 2012-March 2013). We validated SOHCA and sCAHP scores with reference to the original ones in predicting 1-month unfavourable neurological outcomes based on discrimination and calibration measures. Discrimination and calibration were assessed using area under the receiver operating characteristic curve (AUC) and the HosmerLemeshow goodness-of-fit test with calibration plot, respectively.

Results

One-month unfavourable neurological outcome was observed in $82 \%$ of patients. Score availability was significantly higher in the simplified scores than in the original ones and was highest in the sCAHP score (76\%). The AUCs of simplified scores were not significantly different from those of original ones, whereas the AUC of the SCAHP score was significantly higher than that of the sOHCA score ( 0.88 vs. $0.81, P<0.001)$. Goodness-of-fit was poor in the sOHCA score $(v=8, \chi 2=19.1$, Hosmer-Lemeshow test: $P$ $=0.014)$ but not in the SCAHP score $(v=8, \chi 2=13.5$, Hosmer-Lemeshow test: $P=0.10)$.

Conclusion

Performance of original and simplified OHCA and CAHP scores in predicting neurological outcomes in successfully resuscitated OHCA patients were acceptable. Based on the highest availability, similar discrimination, and good calibration, the sCAHP score was the better candidate for clinical implementation. The validated predictive score can help patients' families, healthcare providers, and researchers by accurately stratifying patients.

\section{Background}

Out-of-hospital cardiac arrest (OHCA) is a global medical problem ${ }^{1,2}$. The overall incidence of OHCA is $80-110$ cases per 100,000 population, with approximately 275,000 cases in Europe, 350,000 cases in the US, and 110,000 cases in Japan each year ${ }^{3-5}$. Outcomes after OHCA have improved because of improvements in cardiopulmonary resuscitation (CPR) and treatment strategies. However, even in those who have had successful return of spontaneous circulation (ROSC) and been admitted to hospital for further treatment, outcomes after OHCA remain poor because of post-cardiac arrest syndrome.

Post-cardiac arrest patients often require long-term and expensive life support, resulting in a healthcare burden on families and caregivers. Therefore, prognostication of patients admitted alive is important, as it aids in identifying patients who will benefit most from intensive care, allocating healthcare resources, and counselling patients' families. OHCA ${ }^{6}$ and cardiac arrest hospital prognosis (CAHP) scores ${ }^{7}$ were developed to predict favourable neurological outcomes of patients with ROSC who were admitted to hospital, using variables recorded immediately after admission. The OHCA score is based on measures, including the presence of a shockable rhythm, the no-flow interval (time from collapse to initiation of CPR), the low-flow interval (time from CPR to ROSC), the serum creatinine level, and the arterial lactate level. The CAHP score is based on measures, such as the presence of a shockable rhythm, noflow interval, low-flow interval, total dose of adrenaline (epinephrine) required during CPR, patient's age, arrest setting, and arterial pH.

Both the OHCA and CAHP scores have been validated ${ }^{6-9}$. However, the use of the no-flow interval limits their clinical application, because estimating the time of collapse is quite difficult in unwitnessed OHCA, which account for up to $40 \%$ of $\mathrm{OHCA}^{10,11}$; furthermore, the Utstein template no longer requires no-flow time recording. To address these limitations, Wang et al. ${ }^{12}$ developed the simplified OHCA (sOHCA) score and simplified CAHP (sCAHP) score, in which no-flow time was removed from the measures. They 
reported similar and excellent accuracy of these scores compared to those of the original ones in predicting neurological outcomes in successfully resuscitated OHCA patients.

External validation is an important step before widespread implementation in clinical routine. However, to our knowledge, no study to date has externally validated the SOHCA and SCAHP scores. Since the SOHCA and SCAHP scores were developed based on a singlecentre observational study, validation of these predictive scores based on a distinct multi-centre dataset was warranted. We therefore aimed to externally validate these predictive scores using the Japanese OHCA registry.

\section{Methods}

\section{Study design}

This retrospective observational study evaluated data from the 2012 Survey of Survivors after Out-of-Hospital Cardiac Arrest in the Kanto Region (SOS-KANTO), which was a prospective multicentre observational study in the Kanto Region of Japan that was performed with the support of the Kanto Regional Group of the Japanese Association for Acute Medicine. ${ }^{13}$ Data from adult OHCA patients ( $\geq 18$ years old) who were admitted to hospital after successful ROSC were analysed, although cases were excluded if they involved: (1) extrinsic aetiology (trauma, burn, hypothermia, hanging, drowning, asphyxiation, or drug overdose); or (2) missing data regarding 1-month outcomes after cardiac arrest.

The study's retrospective protocol was approved by the institutional review board of the Tokyo Metropolitan Bokutoh Hospital (approval number: 31-041). All data were de-identified prior to the analysis, and the need for informed consent was waived.

\section{Data collection and outcome measures}

The SOS-KANTO study included 16,452 cardiac arrest patients from 67 emergency medical centres between January 2012 and March $2013 .{ }^{13}$ The emergency medical services (EMS) personnel collected data, including age, sex, bystander-initiated CPR with or without automated external defibrillator (AED) use, cardiac arrest location, witnessed status, initial cardiac rhythm, various time points (e.g., collapse, initiation of CPR, and ROSC), intravenous adrenaline use, and 1-month neurological outcomes, using a form based on the Utstein reporting guidelines. The institutional researchers collected relevant in-hospital information, and neurological outcomes were reported using the cerebral performance category (CPC) scale ${ }^{14}: 1$ (good cerebral performance), 2 (moderate cerebral disability), 3 (severe cerebral disability), 4 (coma or vegetative state), and 5 (death). Cardiac arrest was defined as the cessation of cardiac mechanical activities, confirmed by the absence of pulse and normal breathing. Physicians caring for the patients determined the aetiology of the cardiac arrest.

The original OHCA, SOHCA, original CAHP, and SCAHP scores were calculated based on previous studies ${ }^{6,7,12}$ (see additional file 1). The no-flow interval was defined as the time from collapse to initiation of CPR, and the low-flow interval as the time from initiation of CPR to ROSC. The primary outcome was 1-month unfavourable neurological outcome, defined as CPC 3-5.

\section{Statistical analysis}

For descriptive statistics, numeric variables are presented as medians with interquartile ranges (IQRs) and categorical variables as counts and percentages. All statistical analyses were performed using EZR (Saitama Medical Center, Jichi Medical University, Saitama, Japan $)^{15}$, a graphical user interface for R (The R Foundation for Statistical Computing, Vienna, Austria) ${ }^{16}$ with add-on packages of "PredictABEL" for creating calibration plots and "mice" for multiple imputations. Predictive scores were estimated in each imputed dataset and were integrated based on Rubin's rules. ${ }^{17}$

The external validity of the predictive scores was based on measures of discrimination (ability of the model to discriminate between favourable and unfavourable outcome) and calibration (whether probabilities predicted by the model match observed probabilities). Receiver operating characteristic (ROC) curve analysis was performed, and the area under the ROC curve (AUC) and $95 \%$ confidence intervals $(\mathrm{Cl})$ were used to assess discrimination. The AUC values of original and simplified scores were compared using paired DeLong test. We calculated the optimal cut-off value for predictive scores to distinguish between favourable and unfavourable neurological outcomes. In calculating the cut-off value, we explored both the value that provided the highest combined sensitivity and specificity (Youden Index) and the value that provided positive predictive value (PPV) $>0.99$. Calibration was assessed using the 
Hosmer-Lemeshow goodness-of-fit test. We considered 10 groups in the Hosmer-Lemeshow test. The extent of overestimation or underestimation relative to the observed and predicted rate was explored graphically using calibration plots.

In the main analysis, missing values were handled using the pair-wise deletion method. Additionally, we performed sensitivity analysis using imputed datasets. To obtain approximately unbiased estimates of the parameters, we performed multiple imputations to handle missing data in the analyses. After the above-mentioned inclusion and exclusion criteria were applied, multiple regression, including variables potentially related to missing data (variables listed in Table 1, score availabilities excepted), was conducted. Twenty imputation datasets were generated. Constraint for minimum imputed values was introduced to avoid errors in predictive score calculation. Based on a previous study ${ }^{6}$ and minimum values in the dataset, the lowest possible values for no-flow interval, low-flow interval, lactate, and creatinine were determined as $0.5 \mathrm{~min}, 0.5 \mathrm{~min}, 0.5 \mathrm{mmol} / \mathrm{L}$, and $10 \mu \mathrm{mol} / \mathrm{L}$, respectively.

Table 1

Characteristics of patients who had return of spontaneous circulation and were admitted to hospitals after out-of-hospital cardiac arrest

\begin{tabular}{|c|c|}
\hline Variable & Value \\
\hline Number of patients & 2,428 \\
\hline Patient age, years & $70[60-80]$ \\
\hline Male sex & $1,651(68)$ \\
\hline Cardiac arrest at home & $1,326(55)$ \\
\hline Witnessed arrest & $1,748(72)$ \\
\hline Bystander CPR & $1,024(42)$ \\
\hline AED use by a bystander & $180(7)$ \\
\hline Initial shockable cardiac rhythm & $528(22)$ \\
\hline Response time, min & $7[5-9]$ \\
\hline No-flow interval, min & $10[4-14]$ \\
\hline Low-flow interval, min & $30[16-41]$ \\
\hline $\mathrm{pH}$ & $6.97[6.84-7.13]$ \\
\hline Lactate $(\mathrm{mmol} / \mathrm{L})$ & $153[129-187]$ \\
\hline Creatinine $(\mu \mathrm{mol} / \mathrm{L})$ & $98[80-135]$ \\
\hline Total dose of adrenaline (epinephrine) during CPR, mg & $2[1-4]$ \\
\hline Original OHCA score available & $855(35)$ \\
\hline Simplified OHCA score available & $1,359(56)$ \\
\hline Original CAHP score available & $1,130(47)$ \\
\hline Simplified CAHP score available & $1,834(76)$ \\
\hline 1-month unfavourable neurological outcome & $1,985(82)$ \\
\hline \multicolumn{2}{|c|}{ Data are presented as number (percentage) or median [interquartile range]. } \\
\hline $\begin{array}{l}\text { CPR: cardiopulmonary resuscitation, AED; automated ex } \\
\text { Arrest Hospital Prognosis }\end{array}$ & cardiac arrest; CAHP, Cardiac \\
\hline
\end{tabular}

All statistical tests were two-tailed, and statistical significance was defined by a P-value $<0.05$ or assessed with $95 \% \mathrm{Cl}$. To address missing values for variables, we excluded incomplete cases on an analysis-by-analysis basis. 


\section{Results}

The SOS-KANTO 2012 study evaluated 16,452 patients, and 3,335 patients were admitted to the hospital after ROSC. After 907 patients (784 patients with extrinsic aetiology and 123 patients with unknown outcome) were excluded, a total of 2,428 patients were deemed eligible for the analysis (Fig. 1). The percentages of missing values for the 15 variables varied between $0 \%$ and $43 \%$ (age: $0 \%$, sex: $0 \%$, cardiac arrest location: $2 \%$, witnessed status: $0 \%$, bystander CPR: $0 \%$, AED use by a bystander: $0 \%$, initial shockable cardiac rhythm: $1 \%$, response time: $0 \%$, no-flow interval: $43 \%$, low-flow interval: $18 \%$, pH: $7 \%$, lactate: $24 \%$, creatinine: $12 \%$, total dose of adrenaline during CPR: $0 \%$, and 1-month neurological outcome: $0 \%$ ). In total, 2,606 of 36,420 items (7\%) were missing, and 1,595 of the 2,484 patient records (64\%) were incomplete. Table 1 shows the patients' baseline characteristics. Median age was 70 (IQR: 60-80) years, and $68 \%$ were men. Of the 2,428 patients, 1,985 (82\%) had unfavourable neurological outcome 1 month after OHCA. A summary of the performance measures for the prediction models is presented in Table 2.

Table 2

Performance measures of the prediction models

\begin{tabular}{|c|c|c|c|c|c|c|c|c|}
\hline & \multirow{2}{*}{$\begin{array}{l}\text { Score } \\
\text { availability }\end{array}$} & \multicolumn{2}{|c|}{ AUC (95\% Cl) } & \multicolumn{2}{|l|}{ Model fit } & \multirow[t]{2}{*}{ Sensitivity ${ }^{a}$} & \multirow[t]{2}{*}{ Specificity ${ }^{a}$} & \multirow{2}{*}{$\begin{array}{l}\text { Proportion } \\
\text { of } \\
\text { PPV > } \\
0.99\end{array}$} \\
\hline & & $\begin{array}{l}\text { Complete } \\
\text { case }\end{array}$ & $\begin{array}{l}\text { Imputed } \\
\text { dataset }\end{array}$ & $\begin{array}{l}\text { Complete } \\
\text { case }\end{array}$ & $\begin{array}{l}\text { Imputed } \\
\text { dataset }\end{array}$ & & & \\
\hline \multicolumn{9}{|l|}{ OHCA score } \\
\hline Original & $35 \%$ & $\begin{array}{l}0.83 \\
(0.79- \\
0.86)\end{array}$ & $\begin{array}{l}0.83 \\
(0.81- \\
0.85)\end{array}$ & Good & Poor & 43 & 91 & $2 \%$ \\
\hline Simplified & $56 \%$ & $\begin{array}{l}0.81 \\
(0.77- \\
0.84)\end{array}$ & $\begin{array}{l}0.82 \\
(0.79- \\
0.84)\end{array}$ & Poor & Poor & 34 & 74 & $4 \%$ \\
\hline \multicolumn{9}{|l|}{ CAHP score } \\
\hline Original & $47 \%$ & $\begin{array}{l}0.90 \\
(0.87- \\
0.92)\end{array}$ & $\begin{array}{l}0.92 \\
(0.91- \\
0.94)\end{array}$ & Poor & Poor & 205 & 313 & $5 \%$ \\
\hline Simplified & $76 \%$ & $\begin{array}{l}0.88 \\
(0.85- \\
0.90)\end{array}$ & $\begin{array}{l}0.91 \\
(0.90- \\
0.93)\end{array}$ & Good & Good & 165 & 240 & $13 \%$ \\
\hline
\end{tabular}

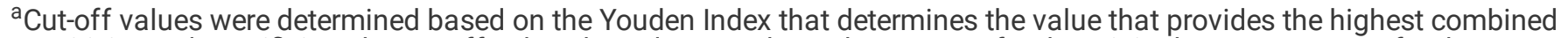
sensitivity and specificity. The cut-off values based on Youden Index were 43 for the original OHCA score, 34 for the sOHCA score, 205 for the original CAHP score, and 165 for the sCAHP score. Goodness-of-fit was assessed using Hosmer-Lemeshow test. A score with $\mathrm{P}>0.05$ in the test was considered to have good calibration.

OHCA, out-of-hospital cardiac arrest; CAHP, cardiac arrest hospital prognosis; AUC, area under the receiver operating characteristic curve; $\mathrm{Cl}$, confidence interval; PPV, positive predictive value

\section{Availability of predictive scores}

Of the 2,428 patients, the sOHCA score was obtained for 1,359 (56\%). Due to one or more missing values in the parameters, the sOHCA score was not available for 1,069 patients.

Of the 1,359 patients whose sOHCA score was obtained, no-flow interval was not recorded for 504 patients, and the original OHCA score was available for 855 (35\% of 2,428) patients. Since the collapse of 395 patients was witnessed, the no-flow interval was inherently unavailable for these patients. Score availability was significantly higher for the SOHCA score than the original OHCA score, with a difference of $21 \%(95 \% \mathrm{Cl}, 18-24 \%)$.

Of all 2,428 patients, sCAHP score was obtained for 1,834 (76\%). Of the 1,834 patients whose sCAHP score was obtained, no-flow interval was not recorded in 704 patients, and the original CAHP score was available for $1,130(47 \%$ of 2,428$)$ patients. No-flow time was unavailable in 529 patients, because the collapse of these patients was not witnessed. Score availability was significantly 
higher for the sCAHP score than the original CAHP score, with difference of $29 \%$ (95\% $\mathrm{Cl}, 26-32 \%)$. When the sOHCA and sCAHP scores were compared, score availability was significantly higher in the sCAHP score, with a difference of $20 \%(95 \% \mathrm{Cl}, 17-22 \%)$.

\section{Discrimination of predictive scores}

Figure 2 shows the ROC curves of predictive scores. The AUC of the original and simplified OHCA scores were $0.83(95 \% \mathrm{Cl}, 0.79-$ $0.86)$ and $0.81(95 \% \mathrm{Cl}, 0.77-0.84)$, respectively. There was no significant difference between the AUC of these two scores $(P=0.41)$.

The AUC of the original and simplified CAHP scores were $0.90(95 \% \mathrm{Cl}, 0.87-0.92)$ and $0.88(95 \% \mathrm{Cl}, 0.85-0.90)$, respectively. There was no significant difference between the AUC of these two scores $(P=0.47)$. Comparing the SOHCA and sCAHP scores, the AUC was significantly higher in the SCAHP score $(P<0.001)$. The cut-off values based on Youden Index and PPV $>0.99$, respectively, were as follows: 43 and 91 for the original OHCA score, 34 and 74 for the sOHCA score, 205 and 313 for the original CAHP score, and 165 and 240 for the sCAHP score. The cut-off values with PPV $>0.99$ gave a false positive rate of $<0.01$ in each score, and identified $14 / 855$ (2\%) patients in the original OHCA score, 58/1359 (4\%) patients in the sOHCA score, 53/1130 (5\%) patients in the original CAHP score, and 241/1834 (13\%) patients in the sCAHP score.

\section{Calibration}

Figure 3 shows the calibration plots, illustrating how the predictive scores performed in predicting 1-month favourable neurological outcome in the study population. The Hosmer-Lemeshow goodness-of-fit test demonstrated that the sOHCA and original CAHP scores had significantly poor model fits $\left(v=8, \chi^{2}=19.1, P=0.014\right.$ and $v=8, \chi^{2}=23.8, P=0.002$; respectively $)$, whereas the original OHCA and SCAHP scores did not $\left(v=8, \chi^{2}=11.1, P=0.196\right.$ and $v=8, \chi^{2}=13.5, P=0.097$; respectively $)$. A calibration plot showed that the sOHCA and original CAHP scores overestimated the probability of the 1-month unfavourable neurological outcome in patients with relatively better prognosis.

\section{Sensitivity analysis}

In the sensitivity analysis, predictive scores were estimated in each of the multiple imputed datasets and were integrated. The AUC of the original and simplified OHCA scores were $0.83(95 \% \mathrm{Cl}, 0.81-0.85)$ and $0.82(95 \% \mathrm{Cl}, 0.79-0.84)$, respectively. The AUC of the original and simplified CAHP scores were $0.92(95 \% \mathrm{Cl}, 0.91-0.94)$ and 0.91 ( $95 \% \mathrm{Cl}, 0.90-0.93)$, respectively. Comparing the sOHCA and sCAHP scores, the AUC was significantly higher in the sCAHP score $(P<0.001)$. The Hosmer-Lemeshow goodness-of-fit test revealed that the original OHCA, original CAHP, and sOHCA scores had significantly poor model fits $\left(v=8, \chi^{2}=72.9, P<0.001 ; v=8\right.$, $\chi^{2}=64.1, P<0.001$; and $v=8, \chi^{2}=33.4, P<0.001$; respectively), whereas $\mathrm{sCAHP}$ score did not $\left(v=8, \chi^{2}=4.0, P=0.86\right)$ (see Additional file 2).

\section{Discussion}

Our analyses showed that both the original and simplified OHCA scores, as well as the CAHP scores, had similar, acceptable discrimination in predicting unfavourable neurological outcome 1 month after OHCA in a Japanese cohort. With the exception of the sOHCA score, these predictive scores also calibrated well. Our findings of acceptable discriminatory performance of the original OHCA and CAHP scores were in agreement with those of previous internal and external validations ${ }^{6-9}$. The result of the sensitivity analysis was largely consistent with these findings. To the best of our knowledge, this is the first study to externally validate the sOHCA and SCAHP scores.

It is desirable for a predictive score to have immediate and wide availability, high discrimination, and good calibration. Both OHCA and CAHP scores are immediately available after routine arterial blood gases are obtained; however, their availability was relatively low in the present study. By removing the no-flow interval from the calculation of these predictive scores, their availability increased 1.6-fold. The proportion of unwitnessed OHCA in our study, $28 \%$, was between the reported range of $11-40 \%{ }^{7-11}$. Since the no-flow interval is inherently unavailable in these unwitnessed OHCA, our results showing significantly higher availability of simplified scores are to be expected. Besides the higher availability, a previous study suggested that removing the no-flow interval from the calculation could have the advantage of improved discrimination, because it decreases the uncertainty caused by estimating the no-flow interval, especially in an area where the proportion of unwitnessed cardiac arrest is relatively high ${ }^{12}$. In the present study, 
discrimination did not improve, but it was maintained. These results indicate that removing the no-flow interval from the calculation is acceptable in terms of discrimination.

A validated predictive score assists clinical decisions and facilitates further clinical research by enabling researchers to compare outcomes between cohorts with different demographics and emergency care systems on a fair basis. Furthermore, validated predictive scores may be considered as a tool to help decide whether further aggressive life-sustaining therapy should be provided. Given that treatment after cardiac arrest costs a great deal and lasts for long periods, an objective and accurate indicator of no chance of favourable neurological outcome could decrease the difficulty for patients' families, as well as healthcare providers. Although there are controversial ethical issues surrounding the futility of the treatment, we explored the cut-off value that provides PPV $>0.99$, based on a traditional definition of medical futility as intervention that provides a $<1 \%$ possibility of favourable neurological outcome ${ }^{18}$. When the cut-off value that provides PPV $>0.99$ was applied to the OHCA patients who had ROSC and were admitted to hospital, the proportion of patients who were considered to have unfavourable neurological outcome was highest in the sCAHP score, in which futility was indicated for $13 \%$ of the patients. These results suggest that the predictive score may enable the identification of futility in the care of OHCA patients after successful ROSC at the very beginning of their treatment in the hospital.

Among the predictive scores assessed, the SCAHP score had the highest availability, and discrimination and goodness-of-fit were comparable to or better than the other scores. Therefore, we believe that SCAHP is the best candidate for clinical implementation. We recognise that prognostication requires caution and should be multimodal whenever possible. Various clinical examinations, electrophysiological measurements, imaging studies, and evaluation of biomarkers have been performed to estimate prognosis after $\mathrm{OHCA}^{19}$. Predictive scores are expected to reinforce the accuracy of these predictive factors, and vice versa. We believe our results showing the validity of predictive scores would help families of patients, healthcare providers, and researchers involved in the care of OHCA patients who have been resuscitated and admitted to hospital.

\section{Limitations}

There are several limitations to the present study. First, the retrospective study design is prone to various biases. The original OHCA score was developed before the study period; thus, it is possible that clinicians used the score during the study period, which possibly affected the results. Although the original CAHP score was described later than the period of the current study, clinicians may have considered some of the factors incorporated into the CAHP score, while making their clinical decisions. Therefore, self-fulfilling prophecy is a potential bias. Second, the reason for missing values was not clear. Although we addressed the missing values using the pair-wise method, this may have biased our results. Third, 1-month CPC grade is a standard assessment of functional outcome after cardiac arrest; however, further studies that take long-term functional outcomes into account are warranted because the functional outcome may change over a long period after cardiac arrest ${ }^{20}$. Fourth, there are no available data regarding the validity of the various measurements in the studied database. Finally, further validation is desirable before implementing a predictive score into practice in a different geographical area, because outcomes after OHCA are related to both patient demographics and EMS services.

\section{Conclusions}

Our analyses externally validated the original OHCA and CAHP, as well as SOHCA and SCAHP scores. By removing the no-flow interval from the calculations, simplified scores increased their availability without significant reduction of discrimination. Among these predictive scores, the SCAHP score demonstrated superior performance; thus, we recommend the use of the sCAHP score in predicting the outcome of patients who are successfully resuscitated and admitted to hospital after OHCA. The use of the SCAHP score is expected to help families of patients, healthcare providers, and researchers by providing accurate stratification of patients.

\section{Abbreviations}

AED, automated external defibrillator; AUC, area under the receiver operating characteristic curve; CAHP, cardiac arrest hospital prognosis; $\mathrm{Cl}$, confidence interval; $\mathrm{CPC}$, cerebral performance category; $\mathrm{CPR}$, cardiopulmonary resuscitation; EMS, emergency medical service; IQR, interquartile range; OHCA, out-of-hospital cardiac arrest; PPV, positive predictive value; ROC, receiver operating characteristic; ROSC, return of spontaneous circulation; SOS-KANTO, Survey of Survivors after Out-of-Hospital Cardiac Arrest in the Kanto Region

Page $7 / 11$ 


\section{Declaratons}

Ethics approval and consent to participate

The study's retrospective protocol was approved by the institutional review board of the Tokyo Metropolitan Bokutoh Hospital (approval number: 31-041). All data were de-identified prior to the analysis, and the need for informed consent was waived.

Availability of data and materials

Registry data is available with permission of the Kanto Regional Group of the Japanese Association for Acute Medicine.

Competing interests

None.

Funding

None.

\section{Acknowledgements}

None.

Authors' contributions

KeS contributed to study conception and design, interpretation of data, statistical analysis, drafting of the manuscript, critical revision of the manuscript for important intellectual content, and provided intellectual input into the research and the manuscript. AS, NK, TT and TN contributed to acquisition of data, critical revision of the manuscript for important intellectual content, and provided intellectual input into the research and the manuscript. KaS, YK, TI, and YH contributed to critical revision of the manuscript for important intellectual content, and provided intellectual input into the research and the manuscript. All authors read and approved the manuscript.

Consent for publication

Not applicable.

\section{References}

1. Sasson C, Rogers MA, Dahl J, Kellermann AL. Predictors of survival from out-of-hospital cardiac arrest: a systematic review and meta-analysis. Circ Cardiovasc Qual Outcomes 2010;3:63-81.

2. McNally B, Robb R, Mehta M, et al. Out-of-hospital cardiac arrest surveillance - Cardiac Arrest Registry to Enhance Survival (CARES), United States, October 1, 2005-December 31, 2010. MMWR Surveill Summ. 2011;60:1-19.

3. Goto Y, Maeda T, Goto YN. Termination-of-resuscitation rule for emergency department physicians treating out-of-hospital cardiac arrest patients: an observational cohort study. Crit Care 2013;17:R235.

4. Benjamin EJ, Blaha MJ, Chiuve SE, et al. Heart Disease and Stroke Statistics-2017 Update: A Report From the American Heart Association. Circulation 2017;135:e146-e603.

5. Berdowski J, Berg RA, Tijssen JG, Koster RW. Global incidences of out-of-hospital cardiac arrest and survival rates: Systematic review of 67 prospective studies. Resuscitation 2010;81:1479-87.

6. Adrie C, Cariou A, Mourvillier B, et al. Predicting survival with good neurological recovery at hospital admission after successful resuscitation of out-of-hospital cardiac arrest: the OHCA score. Eur Heart J. 2006;27:2840-5.

7. Maupain C, Bougouin W, Lamhaut L, et al. The CAHP (Cardiac Arrest Hospital Prognosis) score: a tool for risk stratification after out-of-hospital cardiac arrest. Eur Heart J. 2016;37:3222-8.

8. Hunziker S, Bivens MJ, Cocchi MN, et al. International validation of the out-of-hospital cardiac arrest score in the United States. Crit Care Med. 2011;39:1670-4.

Page 8/11 
9. Isenschmid C, Luescher T, Rasiah R, et al. Performance of clinical risk scores to predict mortality and neurological outcome in cardiac arrest patients. Resuscitation 2019;136:21-9.

10. Matsuyama T, Iwami T, Yamada T, et al. Effect of serum albumin concentration on neurological outcome after out-of-hospital cardiac arrest (from the CRITICAL (2) study in Osaka, Japan). Am J Cardiol. 2018;121:156-61.

11. Lee DH, Cho IS, Lee SH, et al. Correlation between initial serum levels of lactate after return of spontaneous circulation and survival and neurological outcomes in patients who undergo therapeutic hypothermia after cardiac arrest. Resuscitation 2015;88:143-9.

12. Wang $\mathrm{CH}$, Huang $\mathrm{CH}$, Chang WT, et al. Prognostic performance of simplified out-of-hospital cardiac arrest (OHCA) and cardiac arrest hospital prognosis (CAHP) scores in an East Asian population: A prospective cohort study. Resuscitation 2019;137:133-9.

13. SOS-KANTO 2012 Study Group. Changes in treatments and outcomes among elderly patients with out-of-hospital cardiac arrest between 2002 and 2012: A post hoc analysis of the SOS-KANTO 2002 and 2012. Resuscitation 2015;97:76-82.

14. Jacobs I, Nadkarni V, Bahr J, et al. Cardiac arrest and cardiopulmonary resuscitation outcome reports: update and simplification of the Utstein templates for resuscitation registries: a statement for healthcare professionals from a task force of the International Liaison Committee on Resuscitation (American Heart Association, European Resuscitation Council, Australian Resuscitation Council, New Zealand Resuscitation Council, Heart and Stroke Foundation of Canada, InterAmerican Heart Foundation, Resuscitation Councils of Southern Africa). Circulation 2004;110:3385-97.

15. Kanda Y. Investigation of the freely available easy-to-use software 'EZR' for medical statistics. Bone Marrow Transplant. 2013;48:452-8.

16. Team RC. R: a language and environment for statistical computing. R Foundation for Statistical Computing. http://www.Rproject.org/. Accessed 31 October 2018.

17. Rubin DB. Multiple imputation for nonresponse in surveys. New York: J. Wiley \& Sons; 1987.

18. Schneiderman LJ, Jecker NS, Jonsen AR. Medical futility: its meaning and ethical implications. Ann Intern Med. 1990;112:94954.

19. Callaway CW, Donnino MW, Fink EL, et al. Part 8: Post-Cardiac Arrest Care: 2015 American Heart Association Guidelines Update for Cardiopulmonary Resuscitation and Emergency Cardiovascular Care. Circulation 2015;132:S465-82.

20. Tong JT, Eyngorn I, Mlynash M, et al. Functional Neurologic Outcomes Change Over the First 6 Months After Cardiac Arrest. Crit Care Med. 2016;44:e1202-e1207.

\section{Additional Files}

\section{Additional file 1}

File format: .docx

Title of data: Formulae for original and simplified Out-of-Hospital Cardiac Arrest (OHCA) and Cardiac Arrest Hospital Prognosis (CAHP) scores (with permission)

Description of data: ${ }^{\text {a }}$ F, ventricular fibrillation; VT, ventricular tachycardia.

${ }^{\mathrm{b}}$ Natural logarithm of the no-flow interval ( $\left.\mathrm{min}\right)$; lowest possible value $=0.5$.

${ }^{\mathrm{c}}$ Natural logarithm of the low-flow interval (min), lowest possible value $=0.5$.

${ }^{\mathrm{d}}$ Serum creatinine $(\mu \mathrm{mol} / \mathrm{L})$.

e Natural logarithm of plasma lactate $(\mathrm{mmol} / \mathrm{L})$ on admission.

${ }^{\mathrm{f}}$ Total dose of adrenaline during cardiopulmonary resuscitation: 0 points for $0 \mathrm{mg} ; 27$ points for 1 to $2 \mathrm{mg}$; and 43 points for 3 or more mg.

Additional file 2

Page 9/11 
File format: .tif

Title of data: Calibration plots for the predictive scores in predicting 1-month unfavourable functional outcome in the sensitivity analysis

Description of data: Patients were grouped according to 10 percentile increments of predictive probability of unfavourable neurological outcome (cerebral performance category 3-5). The predicted probabilities of unfavourable outcome in each group are plotted against the actually observed proportions. The line shows the points where the predicted and observed values match.

\section{Figures}

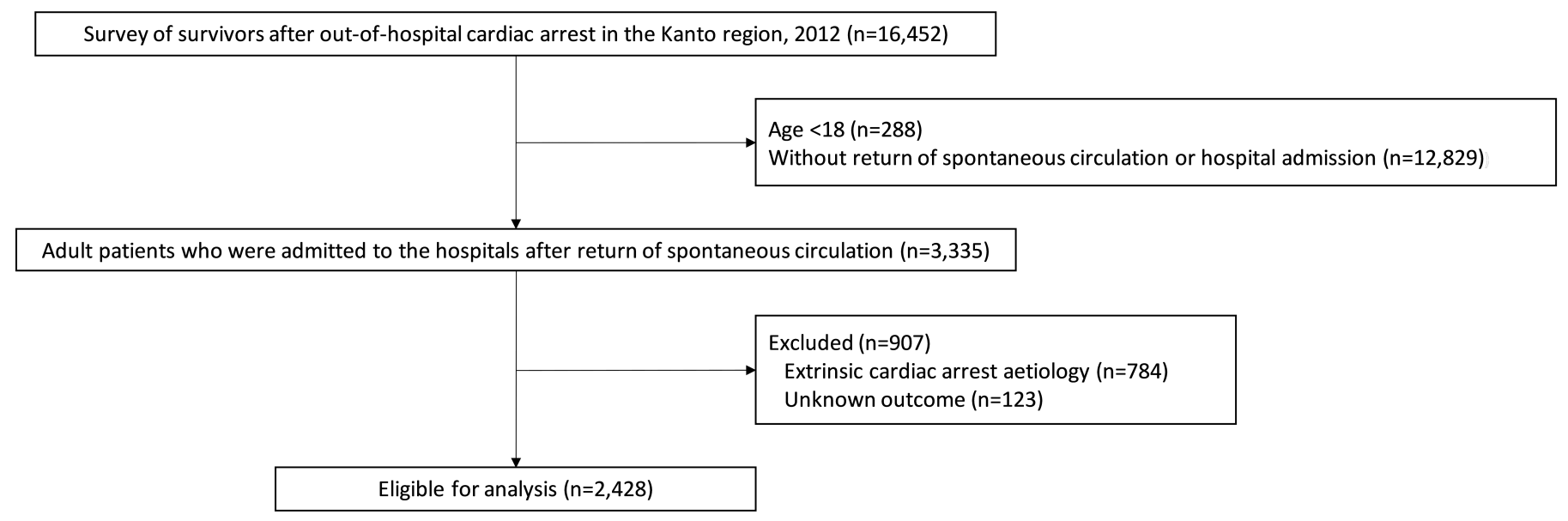

Figure 1

Study flow chart
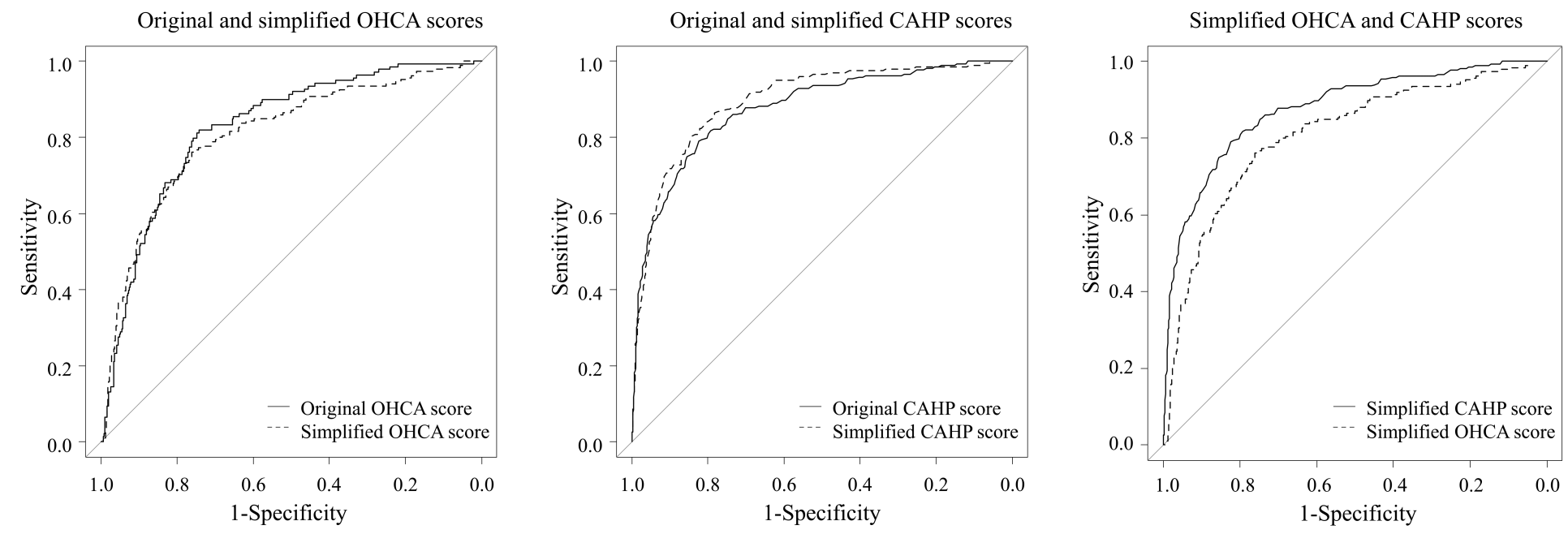

Figure 2

Receiver operating characteristic curve of the predictive scores Left: Receiver operating characteristic curve of the original and simplified OHCA scores. There were no significant differences in area under the curve between the two scores $(P=0.41)$. Middle: 
Receiver operating characteristic curve of the original and simplified CAHP scores. There were no significant differences in area under the curve between the two scores $(P=0.47)$. Right: Receiver operating characteristic curve of the simplified OHCA and simplified CAHP scores. The area under the curve of the simplified CAHP score was significantly higher than that of the simplified OHCA score $(P<0.001)$.
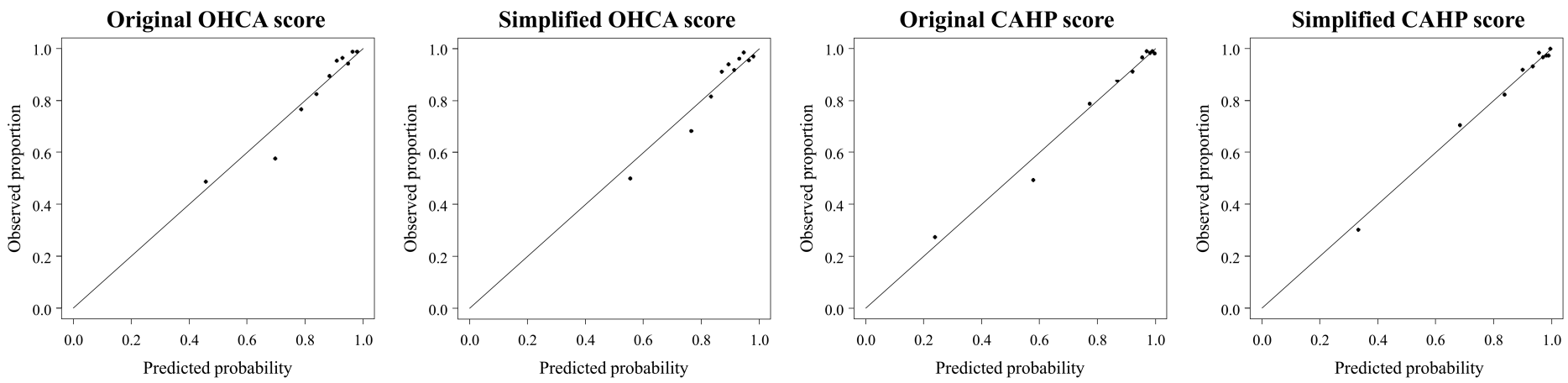

\section{Figure 3}

Calibration plots for the predictive scores in predicting 1-month unfavourable functional outcome Patients were grouped according to 10 percentile increments of predictive probability of unfavourable neurological outcome (cerebral performance category 3-5). The predicted probabilities of unfavourable outcome in each group are plotted against the actually observed proportions. The line shows the points where the predicted and observed values match.

\section{Supplementary Files}

This is a list of supplementary files associated with this preprint. Click to download.

- SupplimentaryFig.1.tif

- SupplementaryTable1.docx 